\title{
Meloxicam combined with sorafenib synergistically inhibits tumor growth of human hepatocellular carcinoma cells via ER stress-related apoptosis
}

\author{
JINGTAO ZHONG $^{1}$, PENG XIU ${ }^{1}$, XIAOFENG DONG $^{2}$, FUHAI WANG $^{1}$, HONGLONG WEI $^{1}$, XIN WANG $^{1}$, \\ ZONGZHEN XU ${ }^{1}$, FENG LIU ${ }^{1}$, TAO LI ${ }^{1}$, YONG WANG ${ }^{1}$ and $\mathrm{JIE} \mathrm{LI}^{1}$ \\ ${ }^{1}$ Department of General Surgery, Qianfoshan Hospital, Shandong University, Jinan, Shandong; \\ ${ }^{2}$ Department of General Surgery, The People's Hospital of Guangxi Zhuang \\ Autonomous Region, Nanning, Guangxi, P.R. China
}

Received June 16, 2015; Accepted July 10, 2015

DOI: $10.3892 /$ or.2015.4181

\begin{abstract}
Sorafenib (SOR) is a promising treatment for advanced hepatocellular carcinoma (HCC). However, the precise mechanisms of toxicity and drug resistance have not been fully explored and new strategies are urgently needed for HCC therapy. Meloxicam (MEL) is a selective cyclooxygenase-2 (COX-2) inhibitor which elicits antitumor effects in human HCC cells. In the present study, we investigated the interaction between MEL and SOR in human SMMC-7721 cells and the role endoplasmic reticulum (ER) stress exerts in the combination of SOR with MEL treatment-induced cytotoxicity. Our results revealed that the combination treatment synergistically inhibited cell proliferation and enhanced apoptosis. Furthermore, the combination treatment enhanced ER stress-related molecules which involved in SMMC-7721 cell apoptosis. GRP78 knockdown by siRNA or co-treatment with MG132 significantly increased this combination treatment-induced apoptosis. In addition, we found that the combination treatment suppressed tumor growth by way of activation of ER stress in in vivo models. We concluded that the combination of SOR with MEL treatment-induced ER stress, and eventually apoptosis in human SMMC-7721 cells. Knockdown of GRP78 using siRNA or proteosome inhibitor enhanced the cytotoxicity of the combination of SOR with MEL-treatment in SMMC7721 cells. These findings provided a new potential treatment strategy against HCC.
\end{abstract}

Correspondence to: Dr Jie Li, Department of General Surgery, Qianfoshan Hospital, Shandong University, 16766 Jingshi Road, Jinan, Shandong, P.R. China

E-mail: lijieqfs@126.com

Key words: hepatocellular carcinoma, meloxicam, sorafenib, combination therapy, endoplasmatic reticulum stress, apoptosis

\section{Introduction}

Hepatocellular carcinoma (HCC) is one of the most common malignant tumors and the fourth primary cause of tumor-related deaths worldwide with high mortality and poor prognosis (1). Although many types of therapeutic measures including surgical resection, transarterial chemoembolization (TACE), radiation and chemotherapy have been used for the treatment of HCC, most patients progress to an advanced stage after the initial therapeutic benefit attributed to high chemoresistance, particularly due to the multidrug resistance (MDR) of HCC (2). Sorafenib (SOR), an oral multikinase inhibitor, which inhibits tumor growth and angiogenesis by way of inhibiting vascular endothelial growth factor receptor 2 and other receptor tyrosine kinases has been used as the standard treatment for advanced stages of HCC based on two large randomized phase III trials, which led to the Food and Drug Administration (FDA) approval since it prolongs survival for 2-3 months in advanced and inoperable HCC cases (3-5). However, clinical results have been disappointing showing that a large number of advanced HCC patients are unresponsive or acquire resistance to SOR. Therefore, it is urgent to seek new effective therapy strategies to combat HCC.

Non-steroidal anti-inflammatory drugs (NSAIDs) have been reported to reduce the risk of developing cancer (6-8). Meloxicam (MEL), a selective cyclooxygenase-2 (COX-2) inhibitor, has been demonstrated to inhibit proliferation and promote apoptosis in many malignant diseases (9-11). Our previous experimental results showed that COX-2 inhibitor exhibits antiproliferative and proapoptotic effects in HCC cell lines $(12,13)$. However, the detailed effects and mechanisms of MEL combined with SOR for treating HCC cells have not been fully cleared. Recently, a number of studies have revealed that certain chemotherapeutics lead to cell death by the way of the ER stress-related apoptosis $(14,15)$. Therefore, we hypothesized that ER stress promoting proapoptotic effects or inhibiting its proliferative function may be a potential target for the treatment of HCC. The endoplasmic reticulum (ER), a central cellular organelle, plays a crucial role in protein folding and maturation as well as accumulation of intracellular calcium. Small 
errors in these processes could disturb normal ER processes and lead to ER stress known as the unfolded protein response (UPR). GRP78, as an ER molecular chaperone, is upregulated when ER stress is induced and functions as a sensory hub and inhibitor of three ER transmembrane receptors: eukaryotic translation initiation factor $2 \alpha$ kinase 3 (EIF2AK3/PERK), inositol-requiring enzyme 1 (IRE1) and activating transcription factor-6 (ATF6) (16). The UPR initially targets proteins for degradation and restores the proper ER homeostasis. However, it eventually induces cell death during intense ER stress (17). Our purpose in the present study was to explore the combined effects of MEL and SOR on apoptosis and evaluate the probable mechanisms of action in HCC cell lines.

\section{Materials and methods}

Cell culture and animals. Human hepatocellular cancer (HCC) SMMC-7721 cells were obtained from the American Type Culture Collection (ATCC; Rockville, MD, USA). The cells were cultured in RPMI-1640 medium (Gibco)/Dulbecco's modified Eagle's medium (DMEM) (HyClone) containing $10 \%$ fetal bovine serum (FBS; Gibco), $100 \mathrm{U} / \mathrm{ml}$ penicillin and $100 \mu \mathrm{g} / \mathrm{ml}$ streptomycin (Gibco) at $37^{\circ} \mathrm{C}$ in $95 \%$ air and $5 \% \mathrm{CO}_{2}$. BALB/c male athymic mice (5-6 weeks old, 18-22 g) were purchased from the Animal Supplier Center of Shandong University. All the animal studies were approved by the Ethics Committee of Shandong University. All surgical procedures were performed under anesthesia with sodium pentobarbital.

Reagents and antibodies. The MEL was purchased from Merck Millipore (Darmstadt, Germany), dissolved in dimethylsulfoxide (DMSO; Sigma-Aldrich, St. Louis, MO, USA) at 10 or $50 \mathrm{mM}$ stock and diluted immediately before each experiment. SOR tosylate was obtained from Bayer Health Care (Berlin, Germany) and dissolved in DMSO to a $10 \mathrm{mM}$ stock. MG132 was obtained from Sigma-Aldrich (San Diego, CA, USA). Primary antibodies to GRP78, caspase-12, PARP and caspase- 3 were purchased from Abcam (Cambridge, UK). Antibodies to IRE1 and phos-eIF2 $\alpha$ were obtained from Cell Signaling Technology (Danvers, MA, USA) and the anti-GAPDH antibody was obtained from Abcam.

Measurement of cell viability. Cell viability assays were performed using the Cell Counting Kit-8 (CCK-8; Dojindo Molecular Technologies, Japan). Cells ( $5 \times 10^{3} /$ well) were seeded with culture medium onto 96 -well plates and incubated at $37^{\circ} \mathrm{C}$ for $24 \mathrm{~h}$. After adaptation, cells were treated with either MEL or SOR, or in combination for $48 \mathrm{~h}$. Then the culture medium was replaced with fresh medium containing $10 \mathrm{ml}$ of CCK-8 solution. The optical density (OD) at $450 \mathrm{~nm}$ was assayed following cell incubation at $37^{\circ} \mathrm{C}$ for $2 \mathrm{~h}$. The viability inhibition rate was calculated as: (control OD value - experiment OD value)/control group OD value x $100 \%$. The coefficient of drug interaction (CDI) analysis for evaluating effects of drug combinations was calculated according to Cao et al (18) using the equation: $\mathrm{CDI}=\mathrm{AB} /(\mathrm{A} \times \mathrm{B})$. $\mathrm{A}$ or $\mathrm{B}$ is the ratio of the single agent group to the control group and $\mathrm{AB}$ is the ratio of the combination groups to the control group. A CDI of $\leq$ or $>1$ indicates synergy, additivity or antagonism, respectively. A CDI $<0.7$ shows that the drugs are significantly synergistic.
Apoptosis assay. Cells were stained with Annexin V-FITC apoptosis detection kit (BD Biosciences, San Jose, CA, USA). According to the manufacturer's instructions, the cells were incubated with $5 \mathrm{ml}$ of Annexin $\mathrm{V}$ and $5 \mathrm{ml}$ of propidium iodide (PI) for $15 \mathrm{~min}$ at room temperature, and then the stained cells were analyzed on a FACS flow cytometer.

Cell cycle analysis by flow cytometry. SMMC-7721 cells were treated with MEL or SOR or in combination for $24 \mathrm{~h}$ and then the cells were performed by cell cycle analysis. In brief, $5 \times 10^{4}$ cells were suspended in $0.5 \mathrm{ml}$ of PI solution, and incubated $30 \mathrm{~min}$ in the dark according to the manufacturer's instructions. Cell cycle distribution was analyzed by FACS flow cytometry.

Cell migration and invasion assays. Cells $\left(1 \times 10^{5}\right)$ in $300 \mathrm{ml}$ of RPMI-1640 medium/DMEM (with 1\% FBS) containing MEL or SOR alone, or in combination were seeded into the upper chamber of a Transwell chamber (Corning, New York, NY, USA). The bottom wells of the chambers were filled with $500 \mathrm{ml}$ RPMI-1640 medium/DMEM containing 10\% FBS. After $48 \mathrm{~h}$ of incubation, the chambers were fixed with $95 \%$ ethanol and then stained with $1 \%$ crystal violet. Similarly, the cell invasion assay was performed by adding Matrigel Basement Matrix to the upper chamber.

Western blot analysis. Western blotting was used to evaluate apoptosis and ER stress-related signaling. After different treatments, protein concentrations in cell extracts were determined (Bio-Rad, Richmond, CA, USA), equal amounts of each sample were resolved in SDS-PAGE gels, then transferred to a polyvinylidene fluoride (PVDF) membrane (Millipore, Billerica, MA, USA), and probed with specific antibodies. Blots were developed using applicable horseradish peroxidase (HRP)-conjugated secondary antibodies and visualized by enhanced chemiluminescence with ECL (Millipore). Protein band intensities were quantified by densitometric analysis using ImageJ software (National Institutes of Health, USA).

Gene transfection and RNAi. For knockdown of GRP78, a small interfering RNA (si-RNA) targeting human GRP78 and a control siRNA were obtained from Santa Cruz Biotechnology (Santa Cruz, CA, USA). SMMC-7721 cells were seeded onto 6-well plates and after $24 \mathrm{~h}$ were transfected using Lipofectamine 2000 transfection reagent (Invitrogen, Carlsbad, CA, USA) according to the manufacturer's instructions. The transfected cells were treated with MEL or SOR or in combination in complete medium for $24 \mathrm{~h}$.

Immunofluorescence assay. Human SMMC-7721 cells seeded onto coverslips in 6-well plates, were fixed with $4 \%$ paraformaldehyde (PFA) and were permeabilized in $0.1 \%$ Triton X-100. Incubation with primary antibodies for $2 \mathrm{~h}$ at room temperature was followed by incubation with fluorescein isothiocyanate (FITC)-labeled IgG secondary antibodies, and then cells were mounted onto microscope slides with a DAPI mounting solution (Abcam). Fluorescent images of the HCC cells were photographed and analyzed with a light microscope (magnification, x200; Olympus, Tokyo, Japan). 
Immunohistochemistry assay. Briefly, tissue sections (4 mm) were deparaffinized in graded xylene and rehydrated in graded ethanol, followed by three washes with phosphate-buffered saline (PBS) for $3 \mathrm{~min}$ each and $1 \% \mathrm{H}_{2} \mathrm{O}_{2}$ for $30 \mathrm{~min}$ in the dark to inhibit endogenous peroxidase activity. Primary anti-caspase-12 (1:500 dilution) antibodies were applied overnight at $4^{\circ} \mathrm{C}$. After washing, the sections were incubated with a biotinylated secondary antibody (Zhongshan, Beijing, China) for $30 \mathrm{~min}$ at $37^{\circ} \mathrm{C}$. Negative control sections were incubated with PBS instead of the primary antibody. The slides were examined under a light microscope (magnification, $x 400$; Olympus).

Measurement of in vivo activity. Under sterile conditions, SMMC-7721 cells $\left(5 \times 10^{6}\right.$ cells/animal) were subcutaneously inoculated into the nude mice. The inoculated mice were randomly divided into four groups, with five mice in each group; the body weight difference between groups was not significant. In the control group, an intraperitoneal injection of saline $(10 \mathrm{ml} / \mathrm{kg})$ was performed every other day. In group 2 and 3, an intraperitoneal injection of MEL $(30 \mathrm{mg} / \mathrm{kg})$ or SOR $(30 \mathrm{mg} / \mathrm{kg}$ ) was administered every other day, respectively. In experimental group 4 (the combination group), MEL $(30 \mathrm{mg} / \mathrm{kg})$ and SOR $(30 \mathrm{mg} / \mathrm{kg})$ were administered by injection every other day. The mice were sacrificed five weeks after tumor implantation. The weights and tumor volumes of the nude mice were recorded every second day until the animals were sacrificed. The tumor volume $(\mathrm{V})$ was monitored by measuring its length (L) and width (W) with calipers and calculated using the following formula: $\mathrm{V}=\left(\mathrm{LxW}^{2}\right) \times 0.5$. The animal care and experimental protocols were in accordance with the institutional guidelines.

Statistical analysis. Data are presented as the mean \pm standard deviation (SD) and were analyzed by one-way ANOVA followed by the Dunnett's test with SPSS software (version 17.0; SPSS China, Shanghai, China), with values of $\mathrm{P}<0.05$ considered to indicate a statistically significant result.

\section{Results}

Combination of MEL and SOR significantly inhibits cell viability in SMMC-7721 cells. In order to investigate the effect of MEL, SOR and their combination on the cell viability of HCC cells in vitro, SMMC-7721 cells were treated with different concentrations of MEL $(0-75 \mu \mathrm{M})$ or SOR $(0-10 \mu \mathrm{M})$. As depicted in Fig. 1, treatment with MEL or SOR for $48 \mathrm{~h}$ significantly inhibited cell viability in SMMC-7721 cells with an $\mathrm{IC}_{50}$ value of $75.6 \pm 0.8 \mu \mathrm{M}$ of MEL alone or an $\mathrm{IC}_{50}$ value of $10.2 \pm 1.5 \mu \mathrm{M}$ of SOR alone. Next, we investigated whether MEL enhanced sensitivity of SMMC-7721 cells to SOR treatment. The CDI was utilized to display the effects of interaction between these two drugs. Our results showed that combination with MEL significantly enhanced SOR lethality and exhibited strong synergistic effects for SMMC-7721 cells (Table I).

Combination of MEL and SOR inhibits migration and invasion in SMMC-7721 cells. Given the association of HCC with a high degree of invasion and metastasis, we investigated whether the migratory potential of SMMC-7721 cells could be

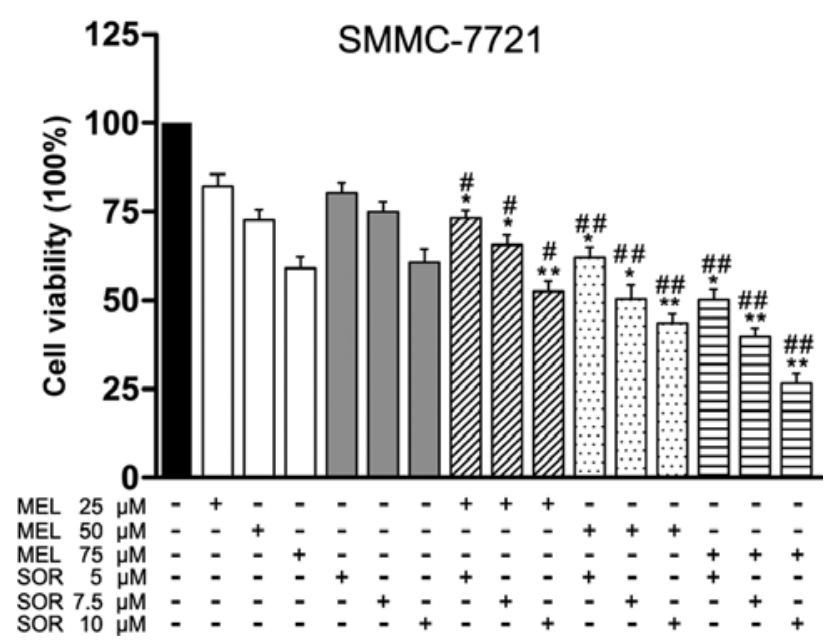

Figure 1. Effect of meloxicam (MEL) and sorafenib (SOR) individually and in combination on the viability of SMMC-7721 cells. Cell vitality was assessed by the CCK-8 assay. SMMC-7721 cells were exposed to MEL and SOR alone or in combination for $48 \mathrm{~h}$. Data are expressed as the percentage of control cells and are the means $\pm \mathrm{SD}$ of three separate experiments. ${ }^{*} \mathrm{P}<0.05 ;{ }^{* *} \mathrm{P}<0.01$ vs. MEL alone, ${ }^{\#} \mathrm{P}<0.05 ;{ }^{\# \#} \mathrm{P}<0.01$ vs. SOR alone.

Table I. CDI of the combination of meloxicam and sorafenib in SMMC-7721 cells.

\begin{tabular}{lcccc}
\hline & & \multicolumn{3}{c}{ SMMC-7721 } \\
& & \multicolumn{3}{c}{ Sorafenib $(\mu \mathrm{M})$} \\
\cline { 2 - 5 } & & 5 & 7.5 & 10 \\
\hline Meloxicam $(\mu \mathrm{M})$ & 25 & 0.893 & 0.838 & 0.734 \\
& 50 & 0.726 & 0.672 & 0.613 \\
& 75 & 0.683 & 0.607 & 0.558 \\
\hline
\end{tabular}

CDI, coefficient of drug interaction.

affected by exposure to MEL or SOR alone or in combination. As determined by scratch motility assay, MEL or SOR treatment alone induced a partial inhibition of migration whereas the combined treatment with MEL and SOR notably inhibited the migratory potential of SMMC-7721 cells (Fig. 2A and C). Furthermore, we applied SMMC-7721 cells to a migration and invasion assay and the results were consistent with those of the scratch assay (Fig. 2B and C).

Combination of MEL with SOR induces cell cycle arrest and apoptosis in SMMC-7721 cells. Given the superior synergistic interactions observed between MEL and SOR, we investigated the potential effects on cell apoptosis mediated by these combinations. Apoptotic cell death induction was analyzed by flow cytometry at $24 \mathrm{~h}$ after SMMC-7721 cells were treatedwith either MEL or SOR alone or in combination. As shown in Fig. 3A and B, MEL and SOR as a single agent led to apoptosis in SMMC-7721 cells. We also observed the MEL+SOR combination significantly increased apoptotic cell death compared with MEL or SOR as a single agent. Next, we used flow cytometry to evaluate the potential effects of MEL and SOR on the cell cycle distribution of SMMC-7721 cells. We 
A

Control

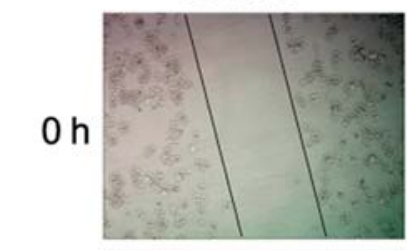

$24 \mathrm{~h}$

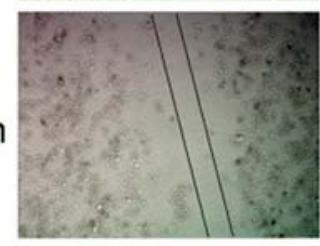

B

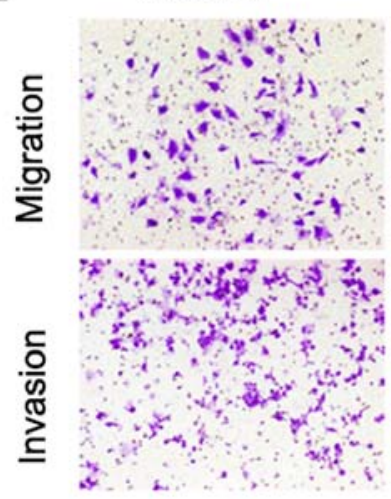

SOR
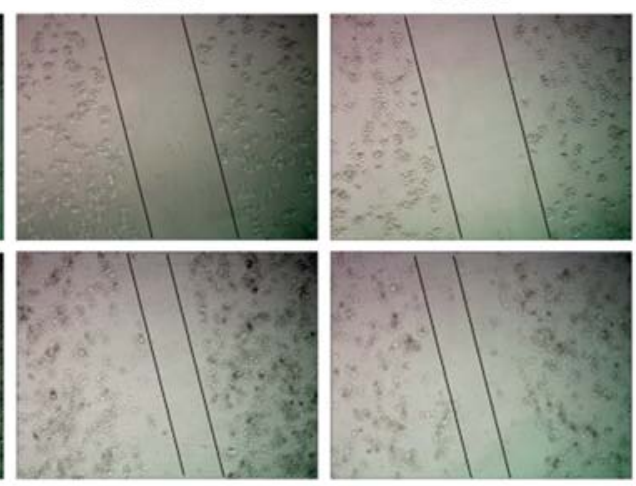

MEL

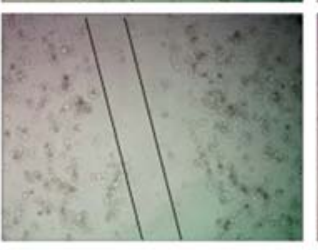

SOR
MEL+SOR

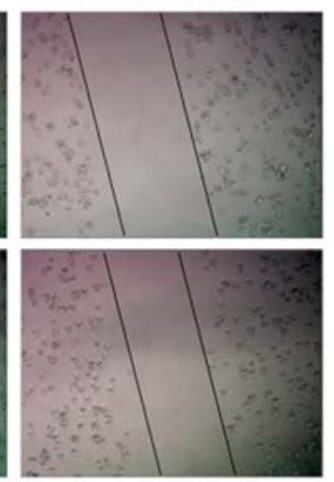

MEL+SOR

C
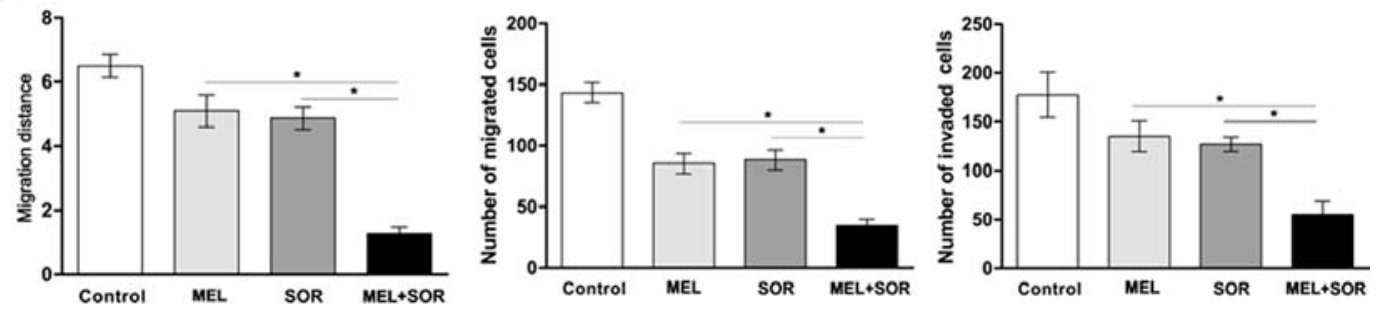

Figure 2. Combination treatment of meloxicam (MEL) and sorafenib (SOR) synergistically reduce migration and invasion ability. (A) Effects of MEL (75 $\mu \mathrm{M}$ ), SOR $(10 \mu \mathrm{M})$ or combined MEL and SOR treatment on the migratory potential of SMMC-7721 cells were analyzed by a scratch assay. Migration was analyzed after $24 \mathrm{~h}$ incubation and photographed (original magnification, $\mathrm{x} 10$ ). Each experiment was carried out in duplicate. (B) Representative images were captured from SMMC-7721 cells incubated with MEL $(75 \mu \mathrm{M})$ and SOR $(10 \mu \mathrm{M})$ either alone or in combination for $48 \mathrm{~h}$ and subjected to cell migration and invasion assays as described in Materials and methods (magnification, x100). (C) The above assays were quantified. Data represent three independent experiments. ${ }^{*} \mathrm{P}<0.01$.

found that cell cycle arrest at the G0/G1 phase was increased with treatment of MEL or SOR compared with the control group (Fig. 3C). In addition, the MEL+SOR combination led to enhanced accumulation of cells in the G0/G1 phase compared to the single agents. These data revealed an additive mechanism of the MEL+SOR combination inducing cell death via apoptosis.

Combination of MEL with SOR induces ER stress in SMMC7721 cells. Previous studies have demonstrated that COX-2 inhibitor and SOR as single agent treatments induced ER stress (19-22). To ascertain whether the MEL+SOR combination treatment enhanced ER stress in HCC cell lines, certain ER-specific signals were measured. Immunoblotting analysis results indicated that the levels of IRE1, p-eIF2 $\alpha$ and GRP78 were upregulated in response to both MEL and SOR alone, and these ER stress marker were significantly increased by the MEL+SOR combination treatment (Fig. 4A and B). To ensure the observations that ER stress-associated markers were increased in SMMC-7721 cells after exposed to MEL or SOR alone or in combination, GRP78 were visualized by immunofluorescence staining. As shown in Fig. 4, immunofluorescence staining of GRP78 was partially increased after MEL or SOR single treatment. However, the combined treatment with MEL and SOR markedly increased GRP78 of SMMC-7721 cells. Several studies have revealed that caspase-12 is activated by continuous ER stress and plays a key role in leading to cell death not via the cytochrome $c$-dependent pathway (23). To investigate the involvement of ER stress in the MEL+SOR combination treatment-induced toxicity and explore potential mechanisms in the present study, western blotting assay was used to detect expression and distribution of caspase-12 proteins in SMMC-7721 cells. As shown in Fig. 4D, the activation of the caspase-12 protein was significantly increased in SMMC-7721 cells that were treated with the MEL+SOR combination compared to the single agents, in agreement with 
A

SMMC-7721
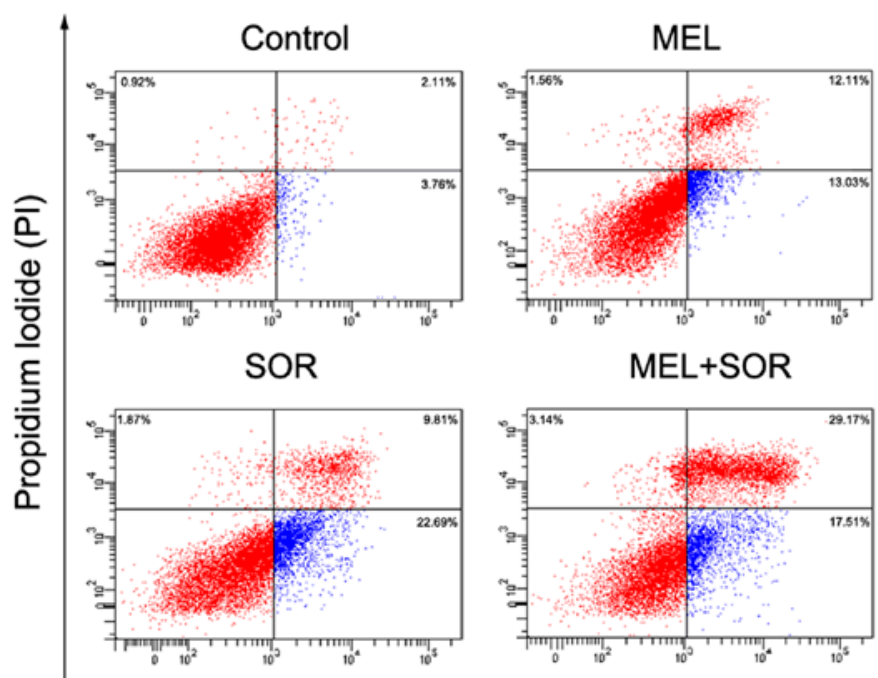

Annexin V-FITC

B

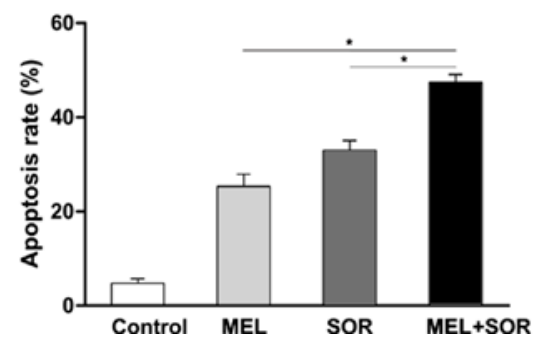

C

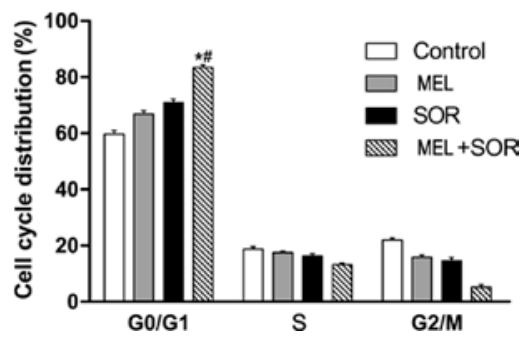

Figure 3. Effect of the treatment with meloxicam (MEL) or sorafenib (SOR) individually and in combination with cell apoptosis and cell cycle distribution of SMMC-7721 cells. (A) Cells were treated with the control (untreated) or MEL $(75 \mu \mathrm{M})$, SOR $(10 \mu \mathrm{M})$ or combined MEL and SOR for $24 \mathrm{~h}$. Apoptotic cells were analyzed by FACS flow cytometry with propidium iodide (PI) and Annexin V-FITC staining. (B) Quantitative analysis of total apoptosis (early and late) population. Data are presented as means \pm SD of three independents experiments. "P $<0.05$. (C) Cell cycle distribution of SMMC-7721 cells was determined $24 \mathrm{~h}$ after treatment with MEL and SOR alone or in combination. Data are expressed as means $\pm \mathrm{SD}$. ${ }^{\mathrm{P}} \mathrm{P}<0.01 \mathrm{vs}$. control, ${ }^{\sharp} \mathrm{P}<0.01 \mathrm{vs}$. MEL or SOR treatment alone.

cell death assays. These data indicated that ER impairment targeted the process of apoptosis.

Involvement of GRP78 in combined MEL with SOR treatment-induced apoptosis. GRP78, one of the most important protective mechanisms induced by UPR has been demonstrated to be associated with chemoresistance (24). In the present study, we explored the role of GRP78 in the MEL+SOR combination treatment-induced apoptosis. As shown in Fig. 5A, transfection of GRP78 siRNA, the MEL+SOR combination treatment significantly reduced cell viability, as expected. Additionally, transfection of GRP78 siRNA which downregulated the level of GRP78 protein (Fig. 5B), notably strengthened the increase of cell apoptosis (Fig. 5C and D) and the cleavage of PARP and caspase-3 (Fig. 5E and F) in the MEL+SOR combination-treated SMMC-7721 cells. These data revealed that GRP78 exerts a protective function in HCC cells to promote drug resistance.

MG132 enhances the MEL and SOR combination treatmentinduced apoptosis in SMMC-7721 cells. Previous studies have revealed that the proteasome pathway exerts a crucial role in the degradation of unfolded protein $(25,26)$. In the present study, we hypothesized that inhibition of proteasome enhances the MEL+SOR combination treatment-induced SMMC-7721 cells apoptosis attributed to the accumulation of unfolded protein. To verify our assumption, the proteasome inhibitor MG132, was used to evaluate the combination effect of the MEL+SOR on human SMMC-7721 cells. Our results showed that when exposed to low-dose $(1 \mu \mathrm{M})$, MG132 mildly affected cell viability. However, the MEL+SOR+MG132 combination treatment significantly enhanced the cell toxicity (Fig. 6A) and apoptosis (Fig. 6B). Furthermore, MG132 significantly suppressed the MEL+SOR combination treatment-induced GRP78 expression and enhanced the cleavage of caspase-3 (Fig. 6C and D). These data revealed that proteosome inhibitor MG132 enhanced the MEL+SOR combination treatment-induced apoptosis.

Combination of MEL with SOR arrests tumor growth in vivo. Due to the superior antitumor effects of the MEL+SOR combination treatment in vitro, we explored whether the MEL+SOR combination treatment inhibited tumor growth in vivo. As shown in Fig. 7A and B, the MEL+SOR 


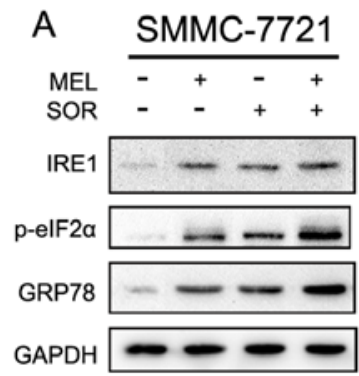

B

C
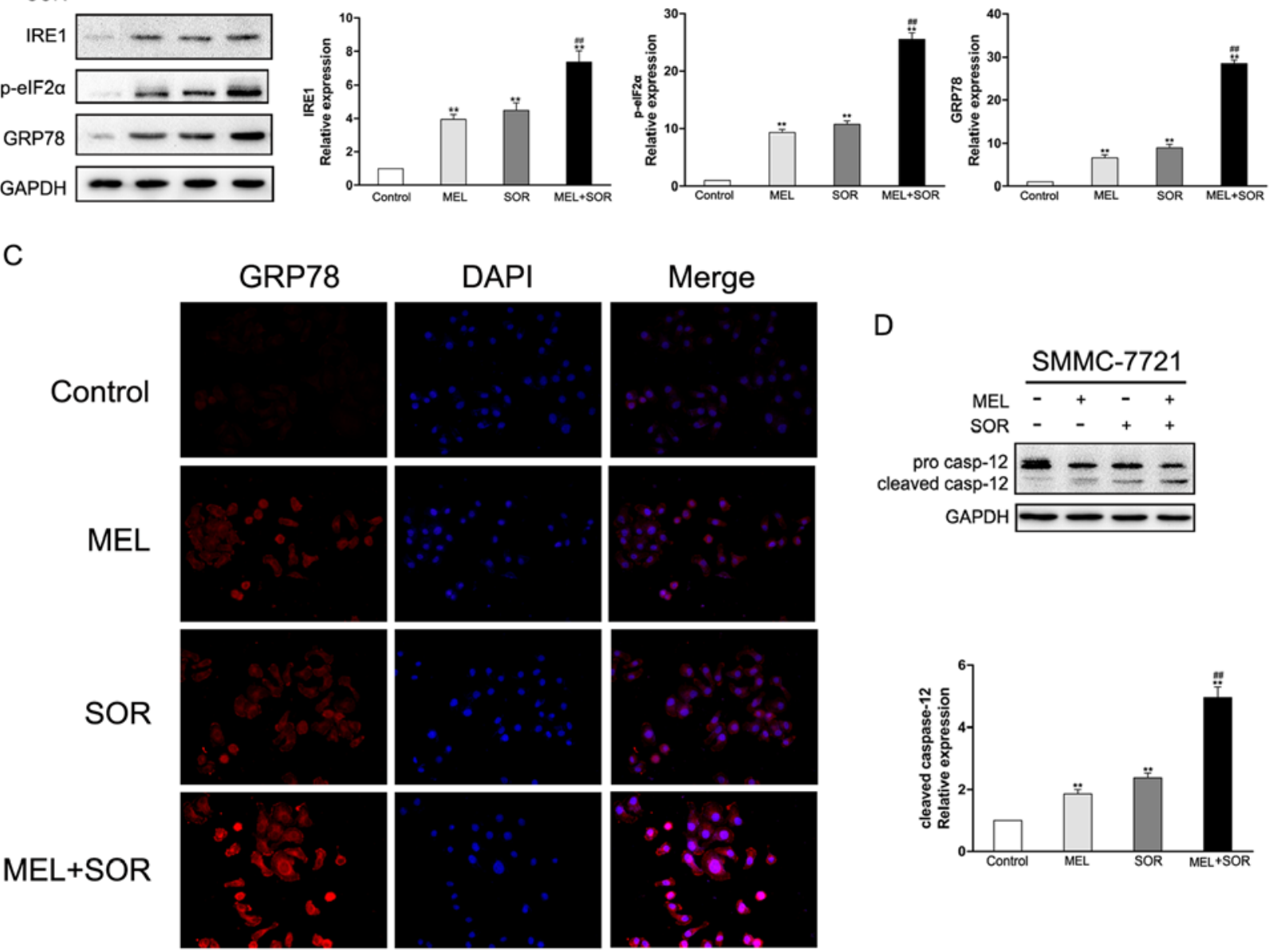

Figure 4. Effect of the treatment with meloxicam (MEL) or sorafenib (SOR) individually and in combination with ER stress-related signaling molecules in SMMC-7721 cells. (A and B) Cells were exposed to the control (untreated) or MEL (75 $\mu \mathrm{M})$, SOR $(10 \mu \mathrm{M})$ or combined MEL and SOR for $24 \mathrm{~h}$. The cell lysates were harvested at $24 \mathrm{~h}$ and analyzed by western blotting with specific antibodies to detect ER stress-related molecules IRE1, p-eIF2 $\alpha$ and GRP78. GAPDH was measured as the loading control. Results shown are representative of at least three independent experiments. ${ }^{* * *} \mathrm{P}<0.01 \mathrm{vs}$. control, ${ }^{\# \#} \mathrm{P}<0.01 \mathrm{vs}$. MEL or SOR alone. (C) Immunofluorescence photomicrography shows expression of GRP78 protein (magnification, x200). (D) Cells were exposed to the control (untreated) or MEL $(75 \mu \mathrm{M})$, SOR $(10 \mu \mathrm{M})$ or combined MEL and SOR for $24 \mathrm{~h}$. The cell lysates were harvested at $24 \mathrm{~h}$ and analyzed by western blotting with specific antibodies to detect caspase-12. GAPDH was measured as the loading control. Results shown are representative of at least three independent experiments. ${ }^{* *} \mathrm{P}<0.01$ vs. control, ${ }^{\# \#} \mathrm{P}<0.01$ vs. MEL or SOR alone. The gels were run under the same experimental conditions.

combination treatment exerted marked antitumor activity in SMMC-7721 xenograft tumors compared to the single agents. However, we found that the combination treatment caused only mild weight change in the in vivo models. Furthermore, we used western blotting and immunohistochemistry to analyze tumor xenografts. The results suggested that the MEL+SOR combination treatment notably activated the ER stress-related apoptosis in SMMC-7721 cell-derived tumors (Fig. 7C and D). In conclusion, our data revealed that the MEL+SOR combination treatment significantly arrests tumor growth in vivo via ER stress-associated regulatory mechanisms.

\section{Discussion}

Hepatocellular carcinoma (HCC), a hypervascular tumor type with characteristic of high levels of neovascularization and angiogenesis, exerts effects in the growth and progression which needs interacting approaches for effective therapy $(27,28)$. Due to the association of single agents with treatment resistance, we considered that the combination therapy increased the lethality in HCC. Sorafenib (SOR) has been applied as the standard therapeutic strategy for advanced HCC patients. In contrast, the selective COX-2 inhibitor has been demonstrated to exert antitumor effects in various types of tumors including $\operatorname{HCC}(12,13,21,29,30)$. Thus, in the present study, we investigated whether the combinations of meloxicam (MEL)+SOR led to more superior antitumor effects than MEL or SOR alone in human SMMC-7721 cells. Our results revealed that either MEL or SOR alone reduce cell viability and colony formation and induce cell cycle arrest and apoptosis. However, the MEL+SOR combination exhibited more potent antitumor effects in terms of cytotoxicity and apoptotic induction via ER stress in human SMMC-7721 cells. GRP78 knockdown by siRNA or proteasome inhibitor significantly enhanced the MEL+SOR combination treatment-induced apoptosis.

ER exerts a key role in regulating protein synthesis, folding and trafficking. A large number of signal pathways have been demonstrated to disrupt the ER function and induce 
A

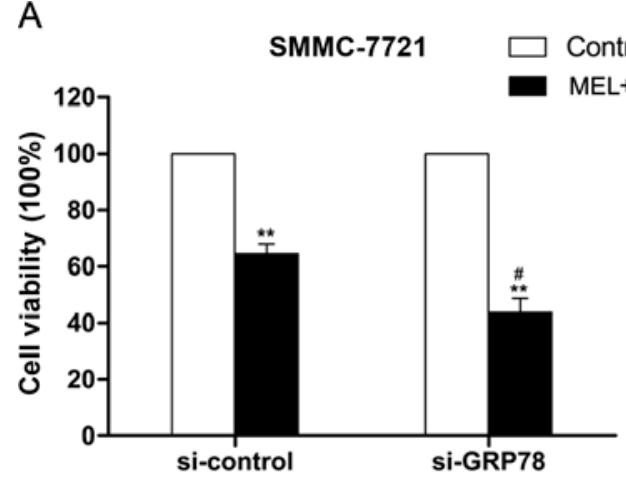

B
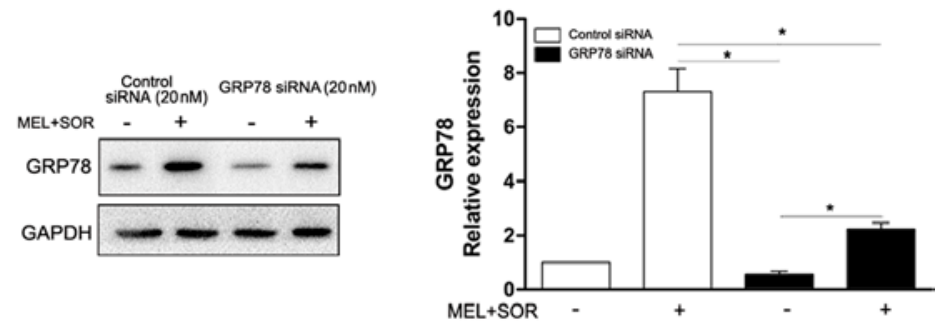

C

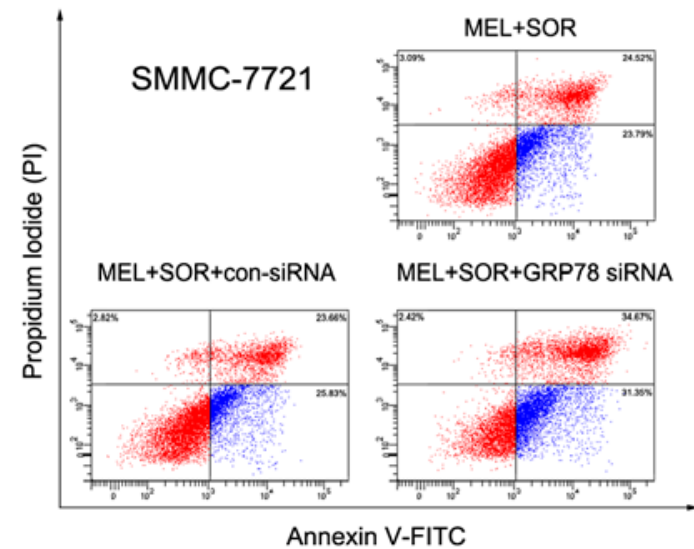

D

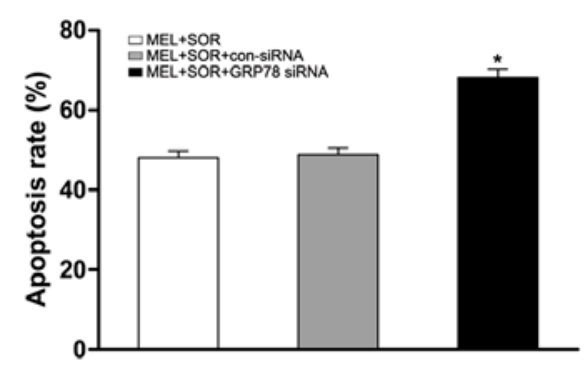

E

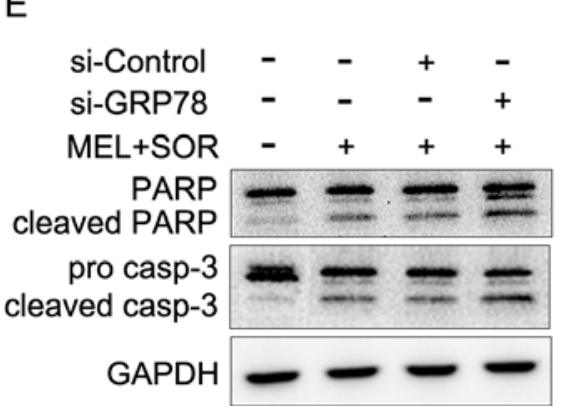

$\mathrm{F}$
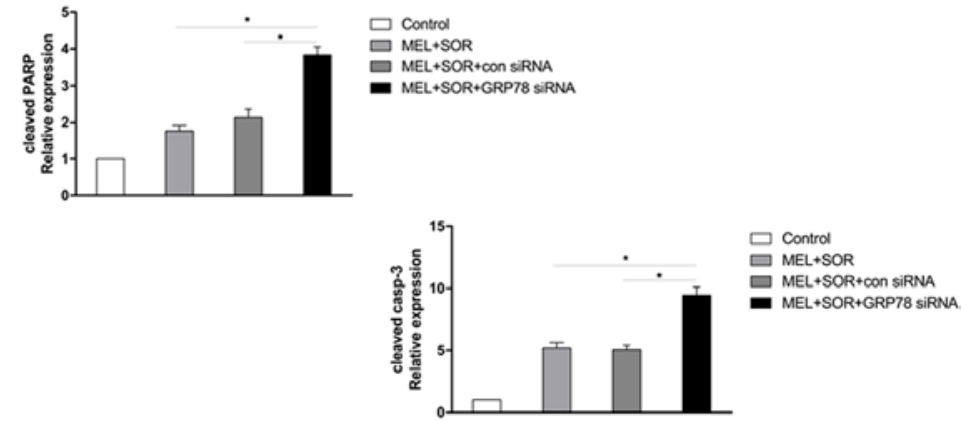

Figure 5. The knockdown of GRP78 significantly enhances the combined effects of meloxicam (MEL) and sorafenib (SOR) on apoptosis of SMMC-7721 cells. (A) Cells were transfected with GRP78 siRNA $(20 \mathrm{nM})$ or scramble siRNA $(20 \mathrm{nM})$ (as a control); then exposed to combined MEL (75 $\mu$ M) and SOR $(10 \mu \mathrm{M})$ or not for $48 \mathrm{~h}$. Data are the means $\pm \mathrm{SD}$ of three separate experiments. ${ }^{* *} \mathrm{P}<0.01$ vs. control, ${ }^{, P} \mathrm{P}<0.05$ vs. MEL+SOR. (B) Cells were transfected with $20 \mathrm{nM}$ GRP78 siRNA and $20 \mathrm{nM}$ scramble siRNA as control, then treated with combined MEL $(75 \mu \mathrm{M})$ and SOR $(10 \mu \mathrm{M})$. Cell lysates were harvested and analyzed by western blotting with specific antibodies against GRP78. GAPDH was measured as the loading control. Results shown are representative of at least three independent experiments. ${ }^{*} \mathrm{P}<0.01$. (C) Cells were transfected with $20 \mathrm{nM}$ GRP78 siRNA and $20 \mathrm{nM}$ scramble siRNA or not (control), then cells were treated with combined MEL $(75 \mu \mathrm{M})$ and SOR $(10 \mu \mathrm{M})$ for $24 \mathrm{~h}$. Apoptotic cells were analyzed by FACS flow cytometry with propidium iodide (PI) and Annexin V-FITC staining. (D) Quantitative analysis of the total apoptotic (early and late) population. Data are presented as the means \pm SD of three independents experiments. "P $<0.05$ vs. control. (E and F) Cells were transfected with $20 \mathrm{nM}$ GRP78 siRNA and $20 \mathrm{nM}$ scramble siRNA or not (control), then cells were treated with combined MEL $(75 \mu \mathrm{M})$ and SOR $(10 \mu \mathrm{M})$. Cell lysates were harvested and analyzed by western blotting with specific antibodies against PAPR and caspase-3. GAPDH was measured as the loading control. Results shown are representative of at least three independent experiments. "P<0.01. The gels were run under the same experimental conditions.

dysfunction of UPR, resulting in ER stress. The initial aim of UPR is to restore ER homeostasis, however, when pro-survival responses failed, these signaling pathways ultimately led to cell apoptosis (14,31-33). GRP78, the ER molecular chaperone, exerts a crucial role in protein folding and assembly (34). Perturbation of ER homeostasis leads to activation of ER stress which results in GRP78 dissociation $(35,36)$. Furthermore, several studies have reported that GRP78 is associated with chemoresistance in cancer therapy $(24,37,38)$. In the present study, the MEL+SOR combination treatment led to ER stress in human SMMC-7721 cells and is associated with the increase of IRE1, p-eIF2 $\alpha$, GRP78 and activation of caspase-12. Silencing GRP78 enhanced the cytotoxic and apoptotic effect of MEL+SOR combination treatment in SMMC-7721 cells. Therefore, it is concluded that GRP78 plays a protective function in HCC cells to promote drug resistance. GRP78 knockdown by siRNA notably increased the susceptibility to MEL+SOR in SMMC-7721 cells. 
A

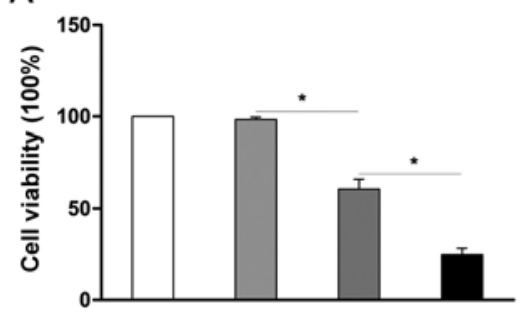

C

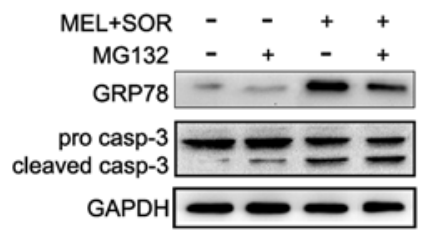

B

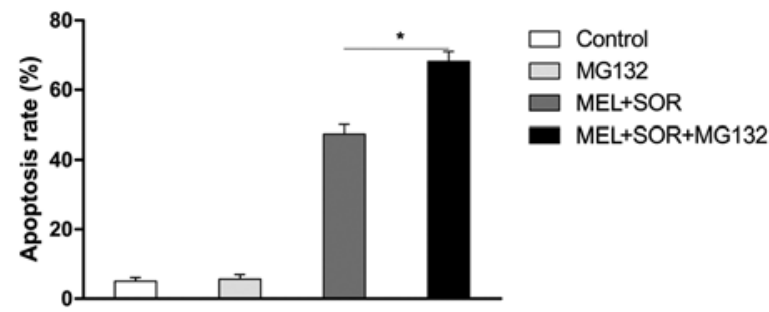

D

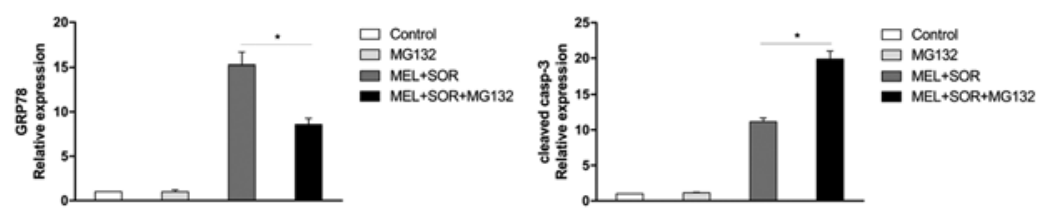

Figure 6. MG132 enhances the meloxicam (MEL) and sorafenib (SOR) combined treatment-induced apoptosis in SMMC-7721 cells. (A) Cell vitality was assessed by the CCK-8 assay. Cells were treated with MEL $(75 \mu \mathrm{M})$, SOR $(10 \mu \mathrm{M})$ and MG132 $(1 \mu \mathrm{M})$ alone or in combination for 48 h. Data are expressed as the percentage of control cells and are the means \pm SD of three separate experiments. ${ }^{*} \mathrm{P}<0.01$. (B) Apoptotic cells were analyzed by FACS flow cytometry with propidium iodide (PI) and Annexin V-FITC staining. Quantitative analysis of the total apoptotic (early and late) population. Data are presented as means \pm SD of three independents experiments. ${ }^{*} \mathrm{P}<0.05$ vs. control. (C and D) Cells were treated with MEL (75 $\left.\mu \mathrm{M}\right)$, SOR $(10 \mu \mathrm{M})$ and MG132 (1 $\left.\mu \mathrm{M}\right)$ alone or in combination for $24 \mathrm{~h}$. Cell lysates were harvested and analyzed by western blotting with specific antibodies against GRP78 and caspase-3. GAPDH was measured as the loading control. Results shown are representative of at least three independent experiments. " $\mathrm{P}<0.01$. The gels were run under the same experimental conditions.

A

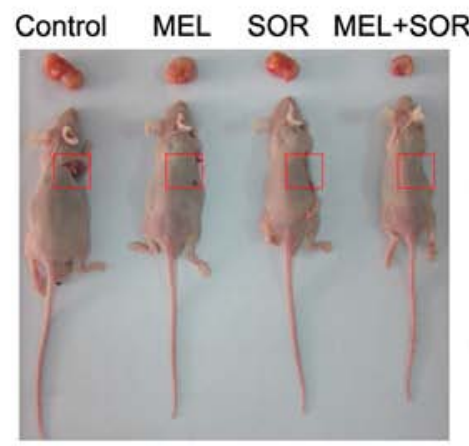

B
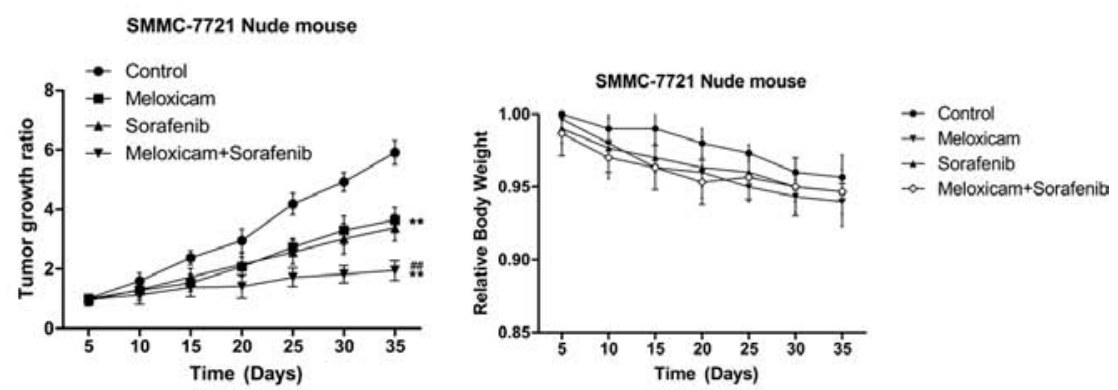

C

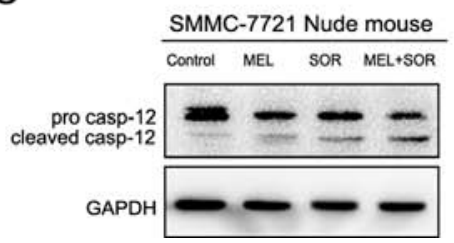

D

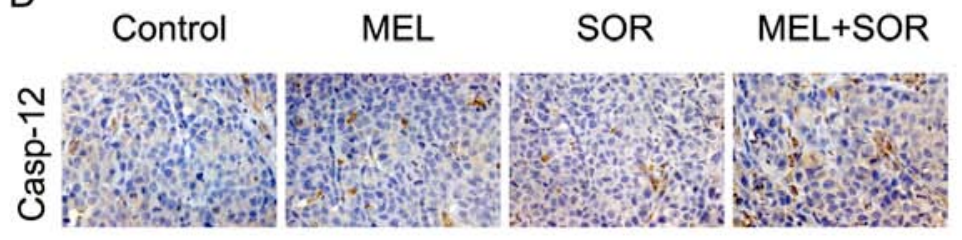

Figure 7. Combination of meloxicam (MEL) with sorafenib (SOR) arrests tumor growth in vivo. (A) Tumors from mice after tumor implantation are shown. Average tumor volume for each group was calculated. (B) Tumor volumes are expressed as the mean \pm SEM. ${ }^{* * *} \mathrm{P}<0.01$ vs. saline-treated controls. ${ }^{\# \#} \mathrm{P}<0.01 \mathrm{vs}$. MEL- or SOR-treated alone. Relative body weights are expressed as the mean \pm SEM. (C) Western blot analysis of SMMC-7721 cell-derived tumors treated with MEL (30 mg/kg) or SOR (30 ml/kg) or combination with expression of caspase-12. GAPDH was measured as the loading control. (D) The expression levels of caspase-12 in SMMC-7721 cell-derived tumors were determined by hematoxylin staining and immunohistochemical analysis (magnification, $\mathrm{x} 400$ ). The gels were run under the same experimental conditions.

Certain studies have revealed that the ubiquitin proteosome pathway exerts a crucial role in intracellular protein degradation by maintaining ER homeostasis when cells encounter the UPR (25). In the present study, our results showed that combined treatment with proteosome inhibitor MG132 significantly enhanced the MEL+SOR-induced cytotoxicity and apoptosis with concomitant downregulation of GRP78 and activation of caspase-3.
In conclusion, these data demonstrated that the MEL+SOR combination treatment notably reduced cell viability and induced apoptosis in human SMMC-7721 cells. GRP78 knockdown or by proteasome inhibitor MG132 significantly enhances the MEL+SOR combination treatment-induced SMMC-7721 cell apoptosis. These findings provide a basis for and warrant future study to investigate the combination of MEL+SOR therapy for the treatment of drug resistant tumors with targeted therapy. 


\section{Acknowledgements}

The present study was supported from the National Natural Scientific Foundation of China (nos. 30972890 and 81172331), the Shandong Provincial Science and Technology Development Planning, China (2010GSF10230), and the Medicine and Health Science Technology of Shandong Province, China (2013WS0145). Thanks to Dr Edward C. Mignot, Shandong University for linguistic advice.

\section{References}

1. Jemal A, Bray F, Center MM, Ferlay J, Ward E and Forman D: Global cancer statistics. CA Cancer J Clin 61: 69-90, 2011.

2. Asghar U and Meyer T: Are there opportunities for chemotherapy in the treatment of hepatocellular cancer? J Hepatol 56: 686-695, 2012.

3. Wilhelm SM, Carter C, Tang L, Wilkie D, McNabola A, Rong H, Chen C, Zhang X, Vincent P, McHugh M, et al: BAY 43-9006 exhibits broad spectrum oral antitumor activity and targets the RAF/MEK/ERK pathway and receptor tyrosine kinases involved in tumor progression and angiogenesis. Cancer Res 64 7099-7109, 2004.

4. Liu L, Cao Y, Chen C, Zhang X, McNabola A, Wilkie D, Wilhelm S, Lynch $\mathrm{M}$ and Carter C: Sorafenib blocks the RAF/MEK/ERK pathway, inhibits tumor angiogenesis, and induces tumor cell apoptosis in hepatocellular carcinoma model PLC/PRF/5. Cancer Res 66: 11851-11858, 2006.

5. Llovet JM, Ricci S, Mazzaferro V, Hilgard P, Gane E, Blanc JF, de Oliveira AC, Santoro A, Raoul JL, Forner A, et al; SHARP Investigators Study Group: Sorafenib in advanced hepatocellular carcinoma. N Engl J Med 359: 378-390, 2008.

6. Thun MJ, Henley SJ and Patrono C: Nonsteroidal anti-inflammatory drugs as anticancer agents: Mechanistic, pharmacologic, and clinical issues. J Natl Cancer Inst 94: 252-266, 2002.

7. Lönnroth C, Andersson M, Asting AG, Nordgren S and Lundholm K: Preoperative low dose NSAID treatment influences the genes for stemness, growth, invasion and metastasis in colorectal cancer. Int J Oncol 45: 2208-2220, 2014.

8. Ding N, Cui XX, Gao Z, Huang H, Wei X, Du Z, Lin Y, Shih WJ, Rabson AB, Conney $\mathrm{AH}$, et al: A triple combination of atorvastatin, celecoxib and tipifarnib strongly inhibits pancreatic cancer cells and xenograft pancreatic tumors. Int J Oncol 44: 2139-2145, 2014.

9. Zhivkova T, Alexandrova R, Dyakova L, Georgieva M, Miloshev G,Culita DC, Marinescu G,Patron L and Alexandrov M: P7.05. Metal complexes of meloxicam and isoxicam decrease viability and proliferation of virus-transformed cancer cells. Ann Oncol 26 (Suppl 2): ii32, 2015.

10. Hassan MH, El-Beshbishy HA, Aly H, Attia SM, Bahashwan SA and Ghobara MM: Modulatory effects of meloxicam on cardiotoxicity and antitumor activity of doxorubicin in mice. Cancer Chemother Pharmacol 74: 559-569, 2014.

11. Arantes-Rodrigues R, Pinto-Leite R, Ferreira R, Neuparth MJ, Pires MJ, Gaivão I, Palmeira C, Santos L, Colaço A and Oliveira P: Meloxicam in the treatment of in vitro and in vivo models of urinary bladder cancer. Biomed Pharmacother 67: 277-284, 2013

12. Li J, Chen X, Dong X, Xu Z, Jiang H and Sun X: Specific COX-2 inhibitor, meloxicam, suppresses proliferation and induces apoptosis in human HepG2 hepatocellular carcinoma cells. J Gastroenterol Hepatol 21: 1814-1820, 2006.

13. Dong X, Li R, Xiu P, Dong X, Xu Z, Zhai B, Liu F, Jiang H, Sun X, Li J, et al: Meloxicam executes its antitumor effects against hepatocellular carcinoma in COX-2-dependent and -independent pathways. PLoS One 9: e92864, 2014

14. Tabas I and Ron D: Integrating the mechanisms of apoptosis induced by endoplasmic reticulum stress. Nat Cell Biol 13: 184-190, 2011.

15. Zhu J, Chen M, Chen N, Ma A, Zhu C, Zhao R, Jiang M, Zhou J, Ye L, Fu H, et al: Glycyrrhetinic acid induces G1-phase cell cycle arrest in human non-small cell lung cancer cells through endoplasmic reticulum stress pathway. Int J Oncol 46: 981-988, 2015.

16. Xu C, Bailly-Maitre B and Reed JC: Endoplasmic reticulum stress: Cell life and death decisions. J Clin Invest 115: 2656-2664, 2005 .
17. Meusser B, Hirsch C, Jarosch E and Sommer T: ERAD: The long road to destruction. Nat Cell Biol 7: 766-772, 2005.

18. Cao SS and Zhen YS: Potentiation of antimetabolite antitumor activity in vivo by dipyridamole and amphotericin B. Cancer Chemother Pharmacol 24: 181-186, 1989.

19. Inamoto $\mathrm{T}$ and Azuma H: Sorafenib increases endoplasmic reticulum (ER) stress in concert with vorinostat. Cancer Biol Ther 12: 1018, 2011.

20. Shi YH, Ding ZB, Zhou J, Hui B, Shi GM, Ke AW, Wang XY, Dai Z, Peng YF, Gu CY, et al: Targeting autophagy enhances sorafenib lethality for hepatocellular carcinoma via ER stressrelated apoptosis. Autophagy 7: 1159-1172, 2011.

21. Suzuki K, Gerelchuluun A, Hong Z, Sun L, Zenkoh J, Moritake T and Tsuboi K: Celecoxib enhances radiosensitivity of hypoxic glioblastoma cells through endoplasmic reticulum stress. Neurooncol 15: 1186-1199, 2013.

22. Kim SJ, Ha GH, Bae JH, Kim GR, Son CH, Park YS, Yang K, Oh SO, Kim SH and Kang CD: COX-2- and endoplasmic reticulum stress-independent induction of ULBP-1 and enhancement of sensitivity to NK cell-mediated cytotoxicity by celecoxib in colon cancer cells. Exp Cell Res 330: 451-459, 2015.

23. Momoi T: Caspases involved in ER stress-mediated cell death. $\mathrm{J}$ Chem Neuroanat 28: 101-105, 2004.

24. Virrey JJ, Dong D, Stiles C, Patterson JB, Pen L, Ni M, Schönthal AH, Chen TC, Hofman FM and Lee AS: Stress chaperone GRP78/BiP confers chemoresistance to tumorassociated endothelial cells. Mol Cancer Res 6: 1268-1275, 2008.

25. Egger L, Madden DT, Rhême C, Rao RV and Bredesen DE: Endoplasmic reticulum stress-induced cell death mediated by the proteasome. Cell Death Differ 14: 1172-1180, 2007.

26. Komatsu S, Miyazawa K, Moriya S, Takase A, Naito M, Inazu M, Kohno N, Itoh M and Tomoda A: Clarithromycin enhances bortezomib-induced cytotoxicity via endoplasmic reticulum stress-mediated CHOP (GADD153) induction and autophagy in breast cancer cells. Int J Oncol 40: 1029-1039, 2012.

27. Dupuy E, Hainaud $P$, Villemain $A$, Bodevin-Phèdre E, Brouland JP, Briand P and Tobelem G: Tumoral angiogenesis and tissue factor expression during hepatocellular carcinoma progression in a transgenic mouse model. J Hepatol 38: 793-802, 2003.

28. Geis T, Döring C, Popp R, Grossmann N, Fleming I, Hansmann ML, Dehne N and Brüne B: HIF-2alpha-dependent PAI-1 induction contributes to angiogenesis in hepatocellular carcinoma. Exp Cell Res 331: 46-57, 2015.

29. Yusup G, Akutsu Y, Mutallip M, Qin W, Hu X, KomatsuAkimoto A, Hoshino I, Hanari N, Mori M, Akanuma N, et al: A COX-2 inhibitor enhances the antitumor effects of chemotherapy and radiotherapy for esophageal squamous cell carcinoma. Int J Oncol 44: 1146-1152, 2014

30. Agarwal V, Hodgkinson VC, Eagle GL, Scaife L, Lind MJ and Cawkwell L: Proteomic (antibody microarray) exploration of the molecular mechanism of action of the specific COX-2 inhibitor DuP 697. Int J Oncol 42: 1088-1092, 2013.

31. Rutkowski DT and Kaufman RJ: A trip to the ER: Coping with stress. Trends Cell Biol 14: 20-28, 2004.

32. Dolai S, Pal S, Yadav RK and Adak S: Endoplasmic reticulum stress-induced apoptosis in Leishmania through $\mathrm{Ca}^{2+}$-dependent and caspase-independent mechanism. J Biol Chem 286: 13638-13646, 2011.

33. Fribley AM, Miller JR, Brownell AL, Garshott DM, Zeng Q, Reist TE, Narula N, Cai P, Xi Y, Callaghan MU, et al: Celastrol induces unfolded protein response-dependent cell death in head and neck cancer. Exp Cell Res 330: 412-422, 2015.

34. Li J and Lee AS: Stress induction of GRP78/BiP and its role in cancer. Curr Mol Med 6: 45-54, 2006.

35. Kulkarni P, Rajagopalan K, Yeater D and Getzenberg RH: Protein folding and the order/disorder paradox. J Cell Biochem 112: 1949-1952, 2011

36. Wu J and Kaufman RJ: From acute ER stress to physiological roles of the Unfolded Protein Response. Cell Death Differ 13: 374-384, 2006.

37. Lee AS: GRP78 induction in cancer: Therapeutic and prognostic implications. Cancer Res 67: 3496-3499, 2007.

38. Ermakova SP, Kang BS, Choi BY, Choi HS, Schuster TF, Ma WY, Bode AM and Dong Z: (-)-Epigallocatechin gallate overcomes resistance to etoposide-induced cell death by targeting the molecular chaperone glucose-regulated protein 78 . Cancer Res 66: 9260-9269, 2006 . 